\title{
Erratum to: The effectiveness of the peer delivered Thinking Healthy Plus (THPP+) Programme for maternal depression and child socio-emotional development in Pakistan: study protocol for A three-year cluster randomized controlled trial
}

\author{
Elizabeth L. Turner ${ }^{1,2}$, Siham Sikander ${ }^{3}$, Omer Bangash $^{3}$, Ahmed Zaidi ${ }^{3}$, Lisa Bates ${ }^{4}$, John Gallis ${ }^{1,2}$, Nima Ganga ${ }^{1}$, \\ Karen O'Donnell ${ }^{1}$, Atif Rahman ${ }^{5^{*}}$ and Joanna Maselko ${ }^{6^{*}}$
}

\section{Erratum}

Unfortunately, the original version of this article [1] contained an error. An error was introduced to the original title of this article.

The title was incorrectly published as:

The effectiveness of the peer-delivered Thinking Healthy PLUS (THPP+) Program for maternal depression and child socioemotional development in Pakistan: study protocol for a randomized controlled trial

The correct title is below:

The effectiveness of the peer delivered Thinking Healthy Plus (THPP+) Programme for maternal depression and child socio-emotional development in Pakistan: study protocol for a three-year cluster randomized controlled trial.

\footnotetext{
Author details

'Duke Global Health Institute, Duke University, Durham, USA. ${ }^{2}$ Department of Biostatistics and Bioinformatics, Duke University, Durham, USA. ${ }^{3}$ Human Development Research Foundation, Islamabad, Pakistan. ${ }^{4}$ Department of Epidemiology, Columbia University School of Public Health, New York, USA. Institute of Psychology, Health and Society, University of Liverpool, Liverpool, UK. ${ }^{6}$ Department of Epidemiology, Gillings School of Public Health, University of North Carolina Chapel Hill, Chapel Hill, USA.
}

Received: 7 October 2016 Accepted: 13 October 2016 Published online: 16 February 2017

\section{Reference}

1. Turner EL, Sikander S, Bangash O, Zaidi A, Bates L, Gallis J, Ganga N, O'Donnell K, Rahman A, Maselko J. The effectiveness of the peer-delivered Thinking Healthy PLUS (THPP+) Program for maternal depression and child socioemotional development in Pakistan: study protocol for a randomized controlled trial. Trials. 2016;17:442.

* Correspondence: atif.rahman@liverpool.ac.uk; jmaselko@unc.edu

${ }^{5}$ Institute of Psychology, Health and Society, University of Liverpool, Liverpool, UK

${ }^{6}$ Department of Epidemiology, Gillings School of Public Health, University of North Carolina Chapel Hill, Chapel Hill, USA 